\title{
Dynamic Spectrum Access in the Time Domain: Modeling and Exploiting White Space
}

Stefan Geirhofer and Lang Tong, Cornell University

Brian M. Sadler, United States Army Research Laboratory

\begin{abstract}
Dynamic spectrum access is a promising approach to alleviate the spectrum scarcity that wireless communications face today. In short, it aims at reusing sparsely occupied frequency bands while causing no (or insignificant) interference to the actual licensees. This article focuses on applying this concept in the time domain by exploiting idle periods between bursty transmissions of multi-access communication channels and addresses WLAN as an example of practical importance. A statistical model based on empirical data is presented, and it is shown how to use this model for deriving access strategies. The coexistence of Bluetooth and WLAN is considered as a concrete example.
\end{abstract}

\section{INTRODUCTION}

Most of the radio spectrum relevant to wireless communications is densely allocated by regulators, making it unlikely that the bandwidth requirements of emerging technologies can be met. Actual measurements illustrate, however, that the scarcity is not a result of heavy usage of the spectrum; in contrast, it is merely due to the inefficiency of the static frequency allocation pursued by regulators. A typical spectrum utilization of around five percent or even less is reported [1].

Dynamic spectrum access (DSA) resolves this paradox by opening assigned, but sparsely used, frequency bands to secondary users, provided that interference to the actual licensee is kept insignificant. Different approaches to this problem were suggested ranging from opening certain bands entirely, in the fashion of the unlicensed industrial, scientific and medical (ISM) bands, to hierarchical schemes requiring secondary users to meet interference constraints [2]. In this article, we restrict ourselves to such hierarchical schemes; that is, we design the cognitive radio so that both systems are orthogonal.

Mutual interference can be avoided by exploiting different degrees of freedom in the design of the secondary system. In particular, orthogonality can be achieved by sufficient spatial separation [3], by orthogonal frequency division multiplexing (OFDM) [4], or by interleaving transmissions in the time domain $[1,5,6]$. The majority of research, so far, has focused on DSA in the spatial domain, meaning that if the primary user's absence in a certain area can be detected reliably, and if we carefully confine our transmissions to this area, interference is limited by this sufficient spatial separation. Reusing TV bands that are allocated, but not broadcast, across the entire country are a prominent example of this paradigm [1].

Dynamic spectrum access is, however, not limited to the spatial domain. As in $[5,6]$, this article aims at achieving orthogonality among users in the time domain by transmitting during the idle periods remaining between the primary user's packet transmissions. Clearly, the problem under consideration is of practical appeal only if the idle periods between packet transmissions are long enough. Although the amount of white space naturally depends on and varies according to the traffic characteristics, our results illustrate that in most cases sufficient white space will be available.

As a motivating example, consider a conference call using the popular voice-over-IP (VoIP) client Skype (with three participating parties). The complex baseband signal for this example is shown in Fig. 1. Although the channel is continuously used for packet transmissions (Fig. 1a), there are large gaps between consecutive packets as illustrated in Fig. 1b. In fact, the channel was idle for 89 percent of the time. The situation is similar for other traffic scenarios $[7,8]$.

There are numerous applications that can take advantage of such transmission opportunities. As an example, consider building automation where sensor networks are used to provide a central controller with local measurements of environment parameters. By the nature of this application, such networks must coexist with 


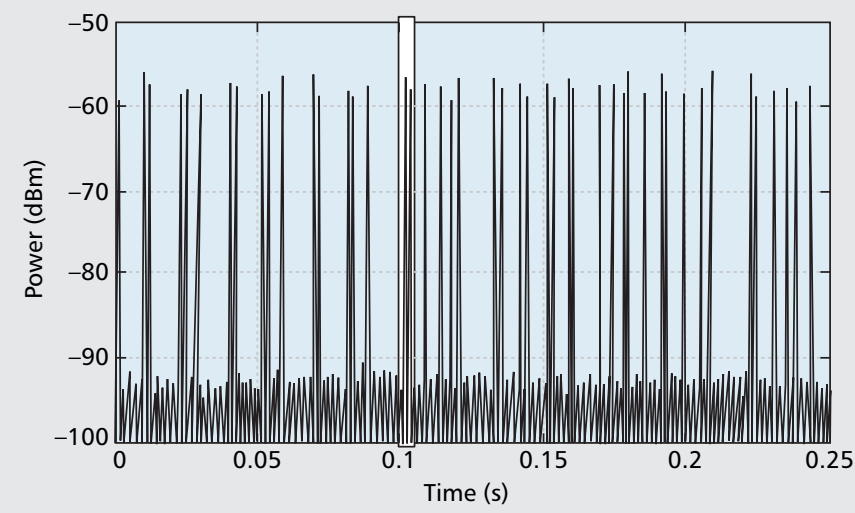

(a)

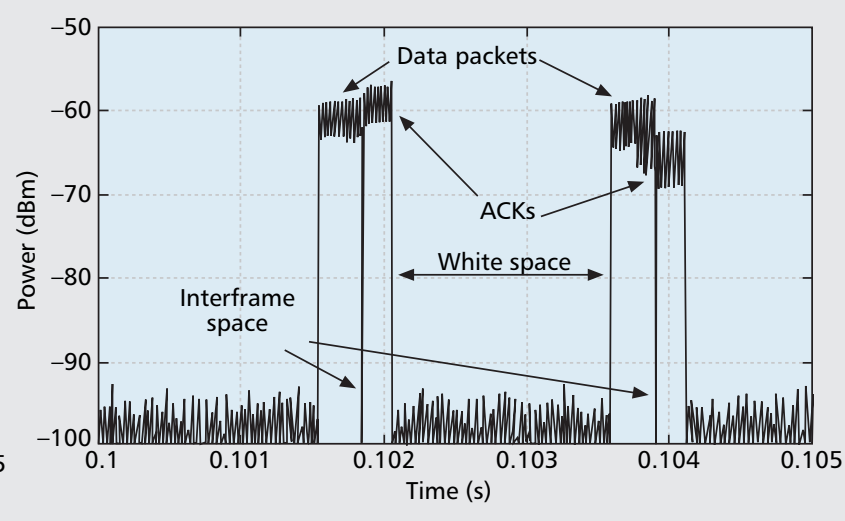

(b)

Figure 1. a) Complex baseband signal of an 802.11b-WLAN supporting a Skype conference call; b) enlarged view of two subsequent packet transmissions.

high-bandwidth data networks (such as WLAN). Given the fact that such sensors communicate only sporadically and at a low rate, it appears reasonable to assume that such systems could efficiently reuse remaining white space.

The existence of sufficient white space raises the question of how to exploit this resource in practice. How complicated is a model that provides adequate prediction performance? Moreover, given a model, how do we apply it to derive practical access schemes for the secondary system? Finally, are such schemes amenable to a real-time implementation, possibly on a batterypowered device with processing limitations?

While some of these questions have been addressed by researchers [1], many issues remain to be investigated. Optimal access strategies were derived in [5] based on partially observable Markov decision processes, assuming that primary and secondary users share the same slotted transmission structure. This assumption, however, is not justified in some practical scenarios. In fact, WLAN transmissions do not have a slot structure but require a continuous channel model.

Finding a realistic yet tractable model to suit such standards is challenging. A bottom-up approach by directly incorporating the specifications of all devices involved is intractable due to the plethora of factors that affect the medium access (number of users, traffic characteristics, higher layer effects, and channel conditions to name only a few). Ultimately, we must strike a balance between statistical accuracy on the one hand, and complexity on the other. We will see that a semi-Markov model is a promising candidate to trade off both extremes [7]. Lastly, we mention the Laycock-Gott model [9] which has been used to predict spectral occupancy in HF bands. Due to different modeling objectives, however, our work requires different statistical techniques.

This article is organized as follows. The experimental test bed used to gather empirical data of the channel's statistics is presented together with possible sensing methods. The main contribution is the establishment of a semi-Markov model; we show how to arrive at estimates for the model parameters and discuss both stationary and nonstationary traffic scenarios. Finally, we give an example of how to apply this model to derive access strategies, focusing on Bluetooth/WLAN coexistence as an example of practical importance.

Throughout the article we identify the primary user with an 802.11 b-based WLAN. We believe this not only is a choice of practical relevance but also can be extended to related multiaccess communications systems.

\section{SENSING Methods: AN EXPERIMENTAL TESTBED}

The reliable detection of primary users is the main challenge in spatial DSA as interference is limited only by sufficient spatial separation. Possible shadowing effects and the hidden-node problem can make it difficult or impossible for a single node to reach the required performance. Cooperative sensing among secondary users becomes indispensable in those cases [10].

For the temporal DSA schemes considered in this article, the sensing burden is alleviated for two reasons. First, we expect to be dealing with a different propagation environment. Most likely we will face an indoor propagation scenario leading to a medium to high signal-to-noise ratio (as is typical for WLAN applications). Second, in temporal DSA, the attention shifts from purely detecting the presence of a primary system to predicting its behavior. We emphasize that it is this prediction - and not the sensing alone that guarantees orthogonality between primary and secondary users. This section briefly outlines the testbed setup used to gather the empirical data our model is based on. Subsequently, two sensing strategies are outlined and their practical relevance is addressed.

\section{Testbed Description}

The data we used to propose and analyze our model was gathered by a testbed consisting of a wireless router and several workstations with WLAN adapter cards (Fig. 2). In contrast to measurement setups considered in other publica- 


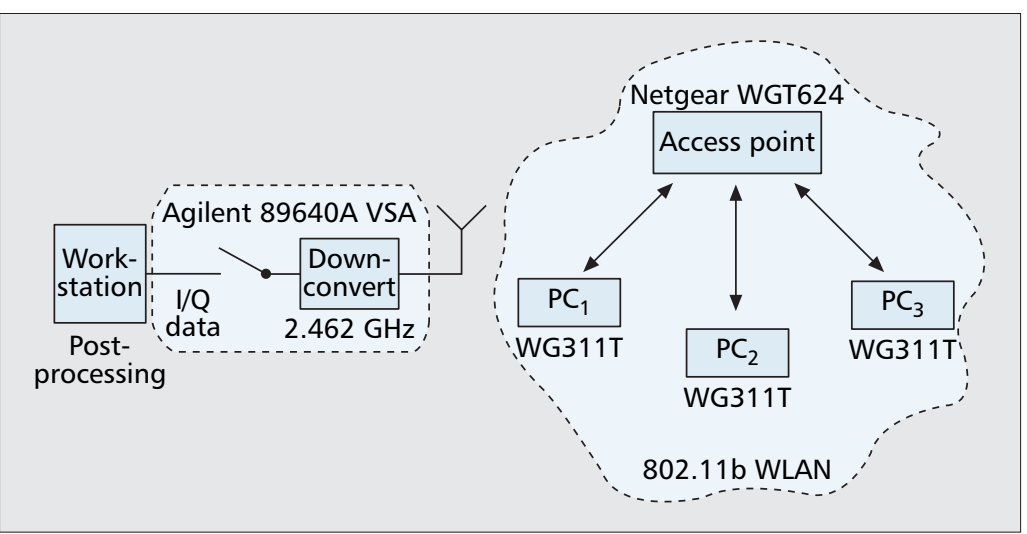

Figure 2. Testbed configuration.

tions, we used a vector signal analyzer to capture the raw complex baseband data. In this way, different sensing strategies can be evaluated on the same data, giving valuable insight when it comes to developing a real-time implementation. Furthermore, by comparing the results of different sensing strategies, the validity of the data could be confirmed.

In addition to the antenna-based setup depicted in Fig. 2, we also tested an isolated setup, where all devices were connected via cables to a resistive power divider. In this way interference from adjacent WLAN hotspots (unrelated to our measurement) could be avoided [7].

We employed different traffic generators to guarantee repeatable measurement results, namely, D-ITG to create synthetic, constant-payload Universal Datagram Protocol (UDP) traffic [7] and GenSyn to generate realistic HTTP traffic [11].

\section{Sensing Strategies}

The strategies for detecting busy and idle periods can be classified according to whether the primary user's transmission standard is known. If not, a logical approach is energy-based detection. On the other hand, if we have information about the primary system, this knowledge can be exploited to achieve better performance. We refer to such methods as feature-based detection.

Energy-based detection is formulated mathematically as discerning the hypotheses of observing only noise and observing a signal in noise, respectively. We assume that a decision is based on a block of complex baseband samples. Given a target value for the error probability, we must choose the number of samples (the block length) such that the specification is met even for the worst case SNR. In practice, if we require an error probability no greater than $10^{-5}$, and if we consider blocks of $1 \mu \mathrm{s}$, a minimum SNR of 4.8 $\mathrm{dB}$ is required. This is a realistic value given usual WLAN setups.

Feature-based techniques improve the detection performance by exploiting standard specifics. In particular, for the 802.11b-based WLAN considered in this work, we can find the start of packets by locking onto the synchronization preamble of the transmitted packets. Moreover, by decoding the LENGTH field within the preamble, we can find the exact packet duration [7].
Although feature-based detection has the ability to increase detection performance, energy detection typically lends itself to a simpler implementation. In practice, additional considerations must be taken into account. For example, feature-based detection has the drawback of requiring a continuous monitoring of the channel. For this reason, energy-based detection should be preferred if the channel is only sensed at certain time instances (e.g., at the beginning of every slot).

\section{MOdeLING WhITE SPACE: A Statistical Approach}

The previous section discussed two sensing strategies for detecting the busy and idle periods of the channel. Based on these data we now develop a statistical model that allows us to predict the channel's behavior. We emphasize that since WLAN does not have a slot structure, a description in continuous time is required. Specifically, our model must incorporate two separate components:

- The states of the channel and their transition behavior

- How long the system resides in each of the states

The previous observation motivates the use of a semi-Markov model, which can be viewed as an extension of a continuous-time Markov chain (CTMC). Although both models describe the transition behavior in the same way, a semiMarkov model allows for specifying the occupancy periods for each state arbitrarily (they need not be exponential as in a CTMC).

\section{Channel State Classification}

In defining the state space, consider again the complex baseband signal shown in Fig. 1b. The figure motivates to extend the state space of the model beyond the trivial busy and idle states. In fact, the WLAN standard specifies that every transmission of a data packet must be followed by an acknowledgment (after a short gap called short inter-frame space (SIFS)). Data packets and acknowledgments serve different purposes and consequently, it makes sense to separate their statistical characterization; ultimately it is very likely for an acknowledgment to follow a data packet. We thus introduce the states DATA, SIFS, and ACK to represent these possible channel conditions (Fig. 3).

First insights into the model can be gained by estimating its transition probabilities. It turns out that the sequence of states DATA $\rightarrow$ SIFS $\rightarrow$ ACK is effectively deterministic, in the sense that the probability of observing this sequence is close to one. This does not come as a surprise since this sequence of states precisely corresponds to a successful packet transmission together with its obligatory acknowledgment. The model thus can be simplified by lumping these three states and defining a new TRANSMIT state that corresponds to such a successful transmission (Fig. 3).

This simplification also makes sense from a practical viewpoint. Ultimately, our model serves the purpose of predicting the idle periods (the white space) of the channel. Although the short 
gap between data packets and their acknowledgments corresponds to an idle channel, it is too short to be used for secondary transmissions.

The previous paragraphs introduced the TRANSMIT state consisting of the transmission of a data packet together with its acknowledgment. Successive transmissions are separated by an idle channel. However, it is important to further differentiate between two cases. First, after a successful transmission, a station cannot continue transmitting but must follow a standardized back-off procedure to give all stations equal access to the medium. This back-off procedure is implemented by a so-called contention period: all stations draw random numbers within a certain range and defer access for this number of slot durations. A station can go ahead and transmit only after having waited for this time period, provided that no other station accessed the medium beforehand. Ideally, this means that the station that draws the smallest number accesses the medium first.

However, not all of the idle periods are due to the contention window. The channel also can be idle because none of the stations has any packets to transmit. In fact, this is precisely the situation we aim to exploit in DSA. We refer to such a channel as being free.

\section{OCCUPANCY DURATIONS}

The simplified model shown in Fig. 3 guides us to the second component of our model: the occupancy duration or sojourn time in each state. To specify this component, we must find (a parametric) distribution that captures this statistical behavior.

The sojourn time in the TRANSMIT state is primarily affected by the traffic characteristics and the scheduler employed in the adapter cards and the wireless router. Transfer of large files generally leads to rather long packets, while streaming traffic such as VoIP leads to shorter packets. From our measurement results for different traffic scenarios, we infer that, in general, several discrete components are observed.

The statistical characterization of the IDLE channel is more challenging since, as mentioned before, the channel can be idle either due to the contention window or a truly free channel. This suggests a mixture distribution, incorporating the effects from both states. Indeed, the contention period shows an almost uniformly distributed sojourn time (ranging from 0 to $0.7 \mathrm{~ms}$ ), while a free state exhibits heavy-tailed behavior that is well approximated by a generalized Pareto distribution.

The empirical distribution function for the idle periods of the channel is shown in Fig. 4 for the case of constant payload UDP traffic. The effects of the mixture-distribution (uniformly distributed body, but heavy tail) are clearly visible. Note the bend in the distribution at approximately $0.7 \mathrm{~ms}$. We also can see that the influence of the contention window becomes more pronounced as the channel gets busier (upper curve). This is intuitive, since the contention procedure is applied more often as traffic load increases.

In addition to this mixture fit, we used phasetype distributions to capture the data statistical-

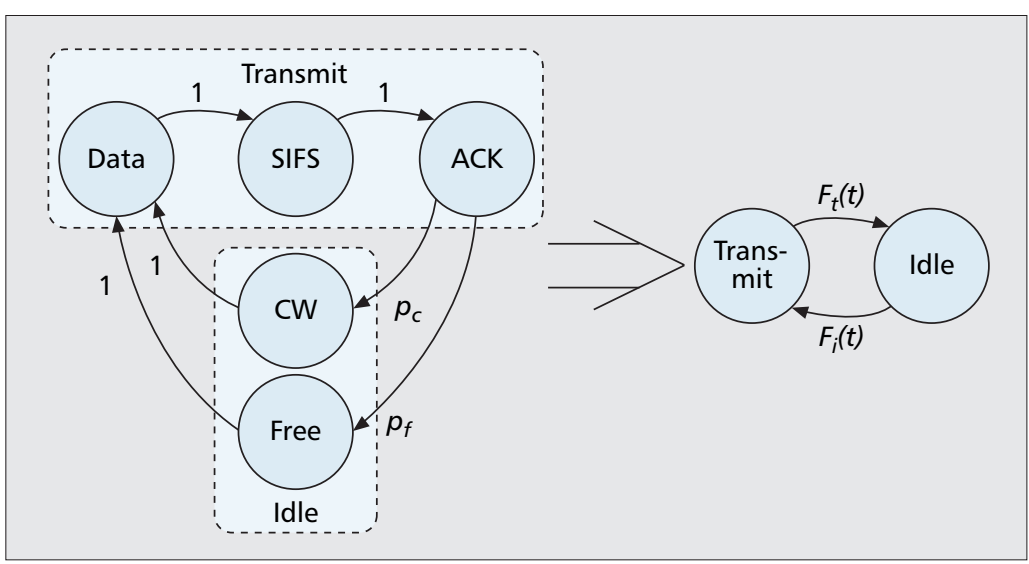

Figure 3. The proposed semi-Markov model.

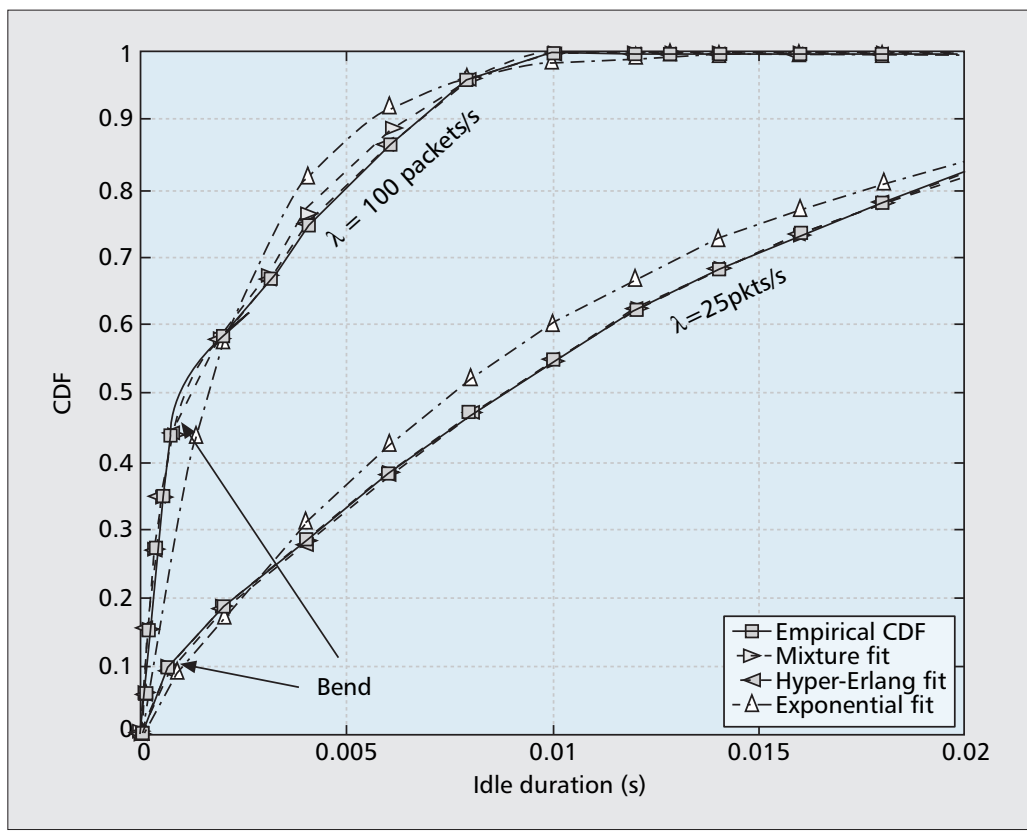

Figure 4. Goodness-of-fit analysis for constant payload UDP traffic.

ly. Such distributions have the advantage that they can be viewed as the time-to-absorption of a CTMC with multiple states, generally allowing for some simplification in applying the model. A hyper-Erlang distribution resulted in a lead to a good fit. Furthermore, an efficient ExpectationMaximization (EM) algorithm exists for estimating the parameters of this model [12]. The fit appears again in Fig. 4.

We also fitted an exponential distribution to the data. Although it does not approximate the empirical data as well as the distributions discussed previously, it makes the model easier to apply in practice. Statistically, such an approach is not entirely justified, but it is worthwhile to identify heuristic algorithms.

\section{GOODNESS-OF-FIT ANALYSIS}

The accuracy of the above fitting methods can be evaluated by looking at the plots in Fig. 4 . Beyond this intuitive assessment, an analytical analysis can be based on the KolmogorovSmirnov (K-S) test, which is a well-established technique in statistics [13]. 


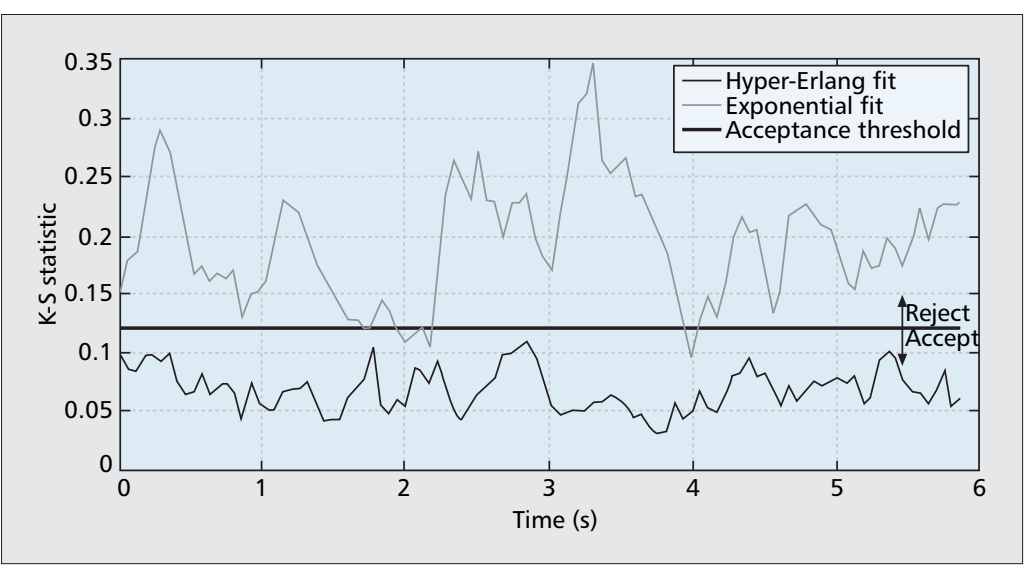

Figure 5. Non-stationary traffic. While the hyper-Erlang fit shows an appropriate goodness of fit, the exponential distribution again exceeds the threshold defined by the $K-S$ test. specified Web pages, but in a way that mimics the behavior of a real user and thus accounts for the non-stationary behavior of HTTP [11].

We again consider a hyper-Erlang distribution and approximate the non-stationary traffic with blocks of length $L$. Within a block the traffic parameters are assumed constant. In choosing $L$, we must strike a balance between approximating the time variations and being able to estimate the model parameters accurately. A good trade-off was obtained for $L=100$.

This approach is illustrated in Fig. 5 for a hyper-Erlang and an exponential fit, respectively. The figure shows how the goodness-of-fit evolves with the time variations of the traffic. Similar to the stationary scenario, the hyper-Erlang distribution shows a satisfactory goodness-of-fit over the entire sample, while the exponential distribution is clearly rejected by the K-S test.

\section{DERIVING ACCESS SCHEMES: A PRACTICAL EXAMPLE}

data is drawn independently from a distribution $F(t)$, namely the one we want to postulate in our model. Intuitively, if this were the case, we would expect the empirical cumulative distribution function $(\mathrm{E}-\mathrm{CDF}) F_{e}(t)$, based on the data alone, to resemble $F(t)$ closely. Indeed, the K-S test is based on the maximum aberration between $F_{e}(t)$ and $F(t)$; a small aberration suggests that the samples come from the proposed distribution.

Although this maximum aberration, also referred to as K-S statistic, reflects the goodnessof-fit quantitatively, it is not normalized by the sample size $N$. Ultimately, if $F_{e}(t)$ is constructed from a large set of observations, we expect a better fit than from a small sample. This is accounted for by the significance level $\alpha$, which specifies the maximum tolerable aberration in relation to $N$ [13]. Usually, a significance level of approximately $\alpha=0.1$ is deemed appropriate.

Stationary UDP Traffic Scenario - We first consider the stationary UDP traffic scenario mentioned previously. The sojourn time in the TRANSMIT state is deterministic due to the constant payload. The statistical distribution for the IDLE state is shown in Fig. 4 for the rates $\lambda$ $=25 \mathrm{pkts} / \mathrm{s}$ and $\lambda=100 \mathrm{pkts} / \mathrm{s}$, respectively. The fact that the traffic is stationary enables us to accurately evaluate the goodness-of-fit based on long trace data.

We can see that both the mixture and the hyper-Erlang distribution show an excellent fit with the E-CDF. The K-S test confirms the accuracy of our model for both distributions. Although Fig. 4 suggests a decent fit for the exponential distribution also, this hypothesis is rejected by the test. Nevertheless, an exponential distribution might still be interesting in practice, because it simplifies the derivation of access schemes.

Non-stationary HTTP Traffic - In the previous stationary traffic scenario, we could evaluate the goodness-of-fit without accounting for time variations. In practice, however, most traffic scenarios are non-stationary. Therefore, we consider more realistic HTTP traffic generated by GenSyn. This traffic generator accesses a list of
In the previous section we proposed a statistical model for the primary user's channel access and verified the goodness-of-fit. Now, we give a concrete example on how to apply this model in practice. Specifically, we show how to enhance the coexistence of Bluetooth and WLAN in the unlicensed ISM band, identifying the primary user with WLAN and the secondary system with Bluetooth.

\section{BLuetooth/WLAN Coexistence}

The coexistence between Bluetooth and WLAN in the unlicensed ISM band is of significant practical concern, because mutual interference can severely limit the performance of both systems [14]. The interference arises from the fact that both standards operate in the ISM band around $2.4 \mathrm{GHz}$, spanning about $80 \mathrm{MHz}$ of bandwidth. The 802.11-based WLAN systems employed today utilize 11 to 13 channels (depending on national standardization), each having a bandwidth of $22 \mathrm{MHz}$. We stress that most of these channels are overlapping and that there is, in fact, only one configuration of three orthogonal channels (Fig. 6). We focus on this configuration in the following paragraphs.

Bluetooth employs frequency-hopping transmissions in $1 \mathrm{MHz}$ wide channels across the same frequency band. In basic operation, the Bluetooth hopping pattern is pseudo-random throughout the entire band and completely oblivious of the WLAN transmissions. Consequently, collisions between WLAN packets and Bluetooth transmissions occur frequently, degrading the performance of both systems.

Given the practical importance of this problem, several propositions on how to mitigate the mutual interference were made. One way of reducing interference is to adapt the hopping sequence of the Bluetooth, such that channels with large interference are avoided. Such methods are referred to as adaptive frequency hopping [14] and consist of the following two main components:

- The sensing and classification of channels according to their interference. 


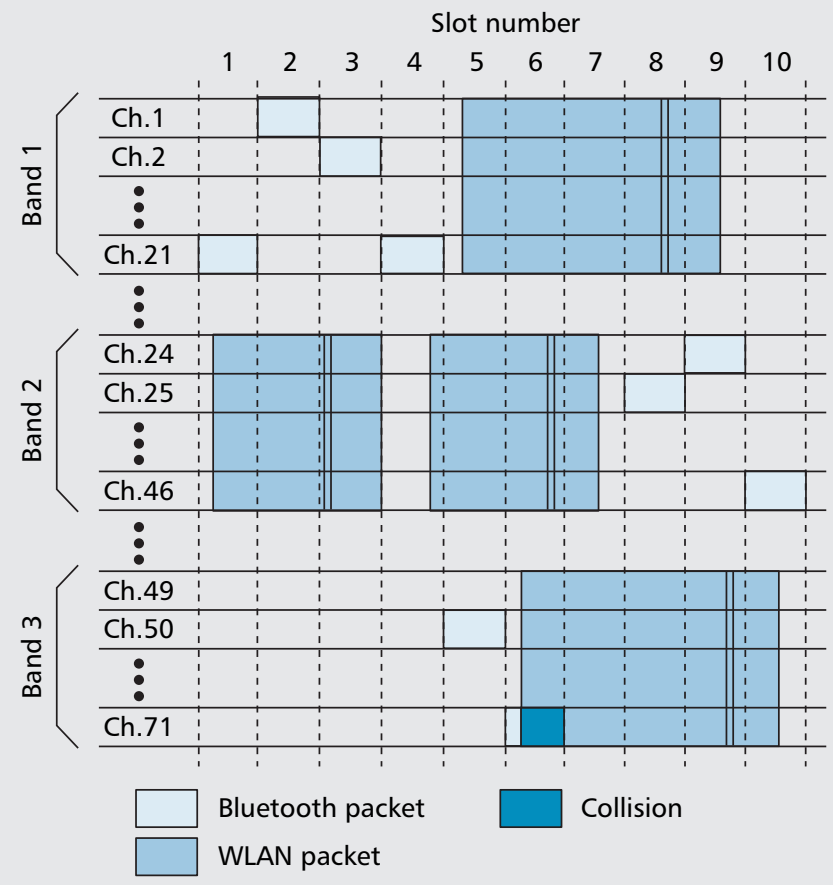

(a)

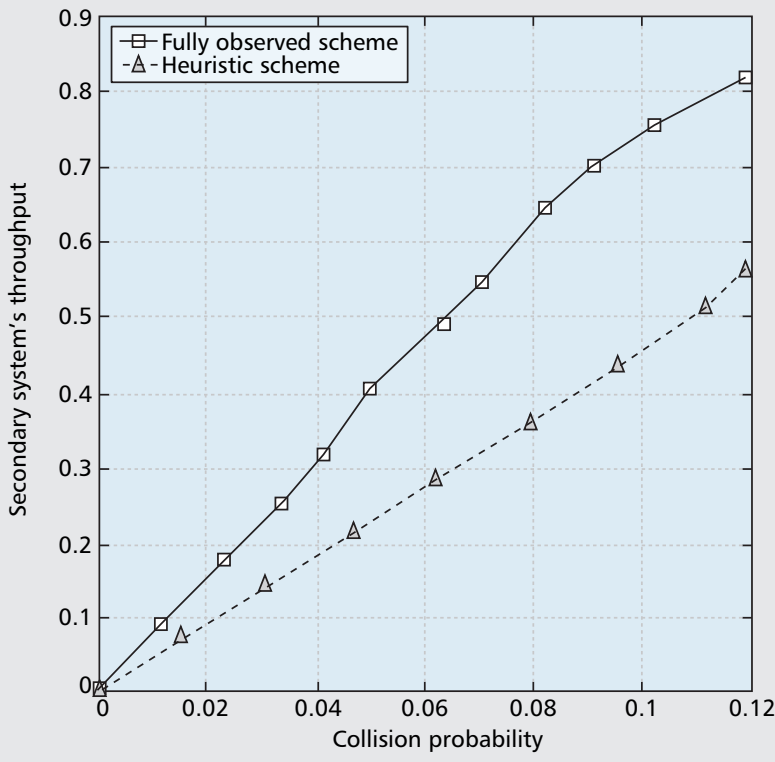

(b)

Figure 6. a) Adaptive frequency hopping; b) performance evaluation. The Bluetooth transmitter preferably hops to bands currently not occupied by the WLAN.

- The adaptation of the hopping sequence such that "bad" channels are avoided whenever possible.

Adaptive frequency hopping was considered previously, based on classifying the Bluetooth channels according to their long-term behavior, for example, by counting the number of bit errors for each channel. Although such an approach can mitigate interference from static sources (e.g., microwave ovens), it is not well suited to capture the time-varying behavior of WLAN interference. In contrast, by incorporating the statistical model proposed in this article, it is possible to predict the behavior of the WLAN and thus to improve the performance significantly.

Our analysis focuses on the representative configuration shown in Fig. 6. There are three orthogonal WLAN channels, each overlapping in frequency with 22 Bluetooth channels. We refer to the WLAN channels as bands to avoid confusion with the Bluetooth channels. Figure 6 illustrates that the Bluetooth transmissions are slotted while WLAN stations can start transmitting at any time. Furthermore, we emphasize that all Bluetooth channels within the same WLAN band show the same access behavior due to the larger bandwidth of the WLAN. Thus it is sufficient to model statistically the behavior of the three WLAN bands rather than each of the 80 Bluetooth channels. The computational burden is thus significantly reduced.

\section{ENHANCED HOPPING SCHEME}

We propose the following adaptive frequencyhopping operation for the Bluetooth transmitter. At the beginning of each slot, the current chan- nel is sensed. If the presence of a WLAN packet is detected, no transmission takes place since this inevitably would lead to a collision. On the other hand, even if no WLAN packet is detected, a collision still can occur if the WLAN becomes active during the subsequent slot (see slot 6 in Fig. 6a). The probability of this event can be kept small, however, by initiating a transmission only with a certain probability $\gamma \leq 1$, even if the channel is sensed idle.

The probability of collision (and thus the design of $\gamma$ ) depends on the WLAN traffic characteristics. In particular, we employ our model to find the probability that conditioned on the channel having been idle for $k$ slots, no WLAN packet will be transmitted during the subsequent slot. By obtaining this probability, we can design $\gamma$ such that collisions with the WLAN occur with a probability smaller than some interference constraint.

The approach described previously constrains the collision probability for each band in Fig. 6 . At the same time, however, we desire to maximize the Bluetooth throughput. This maximization is tightly linked with the optimal hopping across the WLAN bands as each band provides a parallel transmission opportunity. Hardware limitations make it unrealistic that the Bluetooth transmitter can observe the state of all bands at the same time. The problem is thus partially observed, making the optimal design of the system a challenging problem that must be addressed within a decision-theoretic framework $[5,15]$. Although finding the optimal solution to this problem goes beyond the scope of this article, we illustrate the possible performance gain along the lines of a heuristic scheme. 
We emphasize that our model is based on empirical data

gathered from snapshots of the raw complex baseband signal of the WLAN. This enables us to test sensing algorithms on the same data and hence to gain insight into challenges that arise.
Consider a scheme that deterministically switches to the next band whenever the current channel becomes busy. The performance of such a scheme for the UDP traffic scenario considered previously is shown in Fig. 6b. Its performance is compared to a scheme that can observe all of the WLAN bands at the same time. Although this assumption is not necessarily practical, it presents an upper bound on the performance of any partially observed scheme. The figure shows that finding the optimal switching between bands is a worthwhile problem. Ultimately, a possible performance improvement of up to a factor of two can be expected. We also obtained the collision probability and the throughput of a regular (completely oblivious) Bluetooth transmitter. It achieved a (relative) throughput of 70 percent while showing a collision probability of 35 percent. By looking at the performance evaluation in Fig. 6 b, we can see that the regular Bluetooth operation clearly is outperformed.

\section{CONCLUSION}

We addressed the problem of dynamically accessing spectrum in the time domain by taking advantage of white space that remains between bursty packet transmissions of a WLAN. The proposed model statistically captures the medium access of the WLAN but remains tractable enough to be used for deriving practical access schemes for the secondary user. As an example, we have illustrated how our model could be used to enhance the coexistence between WLAN and Bluetooth in the unlicensed ISM band. We emphasize that our model is based on empirical data gathered from snapshots of the raw complex baseband signal of the WLAN. This enables us to test sensing algorithms on the same data and hence to gain insight into challenges that arise when it comes to a realtime implementation.

\section{REFERENCES}

[1] Proc. 1st IEEE Int'l. Symp. New Frontiers in Dynamic Spectrum Access Networks, Nov. 2005

[2] Q. Zhao and B. M. Sadler, "A Survey of Dynamic Spectrum Access: Signal Processing, Networking, and Regulatory Policy," IEEE Sig. Processing, May 2007.

[3] A. Sahai et al., "Fundamental Trade-Offs in Robust Spectrum Sensing for Opportunistic Frequency Reuse," Tech. rep., 2006.

[4] T. A. Weiss and F. K. Jondral, "Spectrum Pooling: an Innovative Strategy for the Enhancement of Spectrum Efficiency," IEEE Commun. Mag., vol. 42, no. 3, Mar. 2004, pp. S8-14.
[5] Q. Zhao, L. Tong, and A. Swami, "Decentralized Cognitive MAC for Dynamic Spectrum Access," Proc. 1st IEEE Int'l. Symp. New Frontiers in Dynamic Spectrum Access Networks, Nov. 2005, pp. 224-32.

[6] P. Papadimitratos, S. Sankaranarayanan, and A. Mishra, "A Bandwidth Sharing Approach to Improve Licensed Spectrum Utilization," IEEE Commun. Mag., vol. 43, no. 12, Dec. 2005, pp. S10-S14.

[7] S. Geirhofer, L. Tong, and B. M. Sadler, "Dynamic Spectrum Access in WLAN Channels: Empirical Model and Its Stochastic Analysis," Proc. 1st Int'l. Wksp. Tech. and Policy for Accessing Spectrum, 2006.

[8] - " "A Measurement-Based Model for Dynamic Spectrum Access," Proc. IEEE MILCOM, 2006.

[9] C. A. Pantjiaros et al., "Development of the LaycockGott Occupance Model," IEE Proc. Commun., vol. 144, no. 1, Feb. 1997, pp. 33-39.

[10] R. Tandra and A. Sahai, "Fundamental Limits on Detection in Low SNR Under Noise Uncertainty," Proc. Int'l. Conf. Wireless Networks, Commun. and Mobile Comp., 2005.

[11] P. E. Heegaard, "GenSyn - A Java Based Generator of Synthetic Internet Traffic Linking User Behavior Models to Real Network Protocols," Proc. ITC Specialist Seminar on IP Traffic Measurement, Modeling, and Management, 2000.

[12] A. Thümmler, P. Buchholz, and M. Telek, "A Novel Approach for Fitting Probability Distributions to Real Trace Data with the EM Algorithm," Proc. Int'l. Conf. Dependable Sys. and Networks, June 2005, pp. 712-21.

[13] R. B. D'Agostino and M. A. Stephens, Goodness-of-Fit Techniques, Marcel Dekker, 1986.

[14] N. Golmie, N. Chevrollier, and O. Rebala, "Bluetooth and WLAN Coexistence: Challenges and Solutions," IEEE Trans. Wireless Commun., vol. 10, no. 6, Dec. 2003, pp. 22-29.

[15] Q. Zhao et al., "Optimal Dynamic Spectrum Access via Periodic Channel Sensing," Proc. IEEE Wireless Commun. and Net. Conf., Mar. 2007.

\section{BIOGRAPHIES}

STEFAN GEIRHOFER [S'05] (sg355@cornell.edu) received a Dipl.-Ing. degree in electrical engineering from the Vienna University of Technology, Austria, in 2005. He is now working toward a Ph.D. in the Adaptive Communications and Signal Processing Group at Cornell University, Ithaca, New York. His research interests focus on signal processing and rapid prototyping in wireless communications, including cognitive radio, dynamic spectrum access, and MIMO systems.

LANG TONG [S'87, M'91, SM'01, F'05] (It35@cornell.edu) is the Irwin and Joan Jacobs Professor in Engineering at Cornell University, Ithaca, New York. He received the 2004 IEEE Signal Processing Best Paper Award and the 2004 Leonard G. Abraham Prize Paper Award. His research is in the general area of statistical signal processing, wireless communications and networking, and information theory.

BRIAN M. SADLER [M'90, SM'02, F'06] (bsadler@arl.army.mil) is a senior research scientist at the Army Research Laboratory, Adelphi, Maryland. He has been an editor for several journals in communications and signal processing. His research interests include signal processing for mobile wireless and ultra wideband systems, and sensor signal processing and networking. 